\title{
Gadolinium Enhancement of the Aneurysm Wall in Extracranial Carotid Artery Aneurysms
}

(D) C.J.H.C.M. van Laarhoven, (D) M.L. Rots, (D) V.E.C. Pourier, (D)N.K.N. Jorritsma, (D)T. Leiner, (D). Hendrikse, (D) M.D.I. Vergouwen, and (D.G.J. de Borst

\begin{abstract}
BACKGROUND AND PURPOSE: The natural history and optimal treatment of extracranial carotid artery aneurysms are unknown. Gadolinium enhancement of the aneurysm wall may reflect aneurysm wall inflammation and instability. In this study, we investigated the feasibility of extracranial carotid artery aneurysm wall imaging and explored a potential relationship of aneurysm wall enhancement with aneurysm growth and the presence of (silent) brain infarcts and white matter lesions.
\end{abstract}

MATERIALS AND METHODS: Fourteen conservatively treated patients with 15 asymptomatic extracranial carotid artery aneurysms underwent gadolinium-enhanced 3T MR imaging at 2 time points with a 12-month interval. Primary outcome was growth of the aneurysm sac ( $\geq 2.0 \mathrm{~mm}$ ); secondary outcomes were the presence of (silent) brain infarcts and white matter lesions at baseline and follow-up. MR images were reviewed by 2 independent observers, and inter- and intraobserver reproducibility was assessed.

RESULTS: Seven (50\%) patients were men; the median age was 55 years (range, 40-69 years). Eleven extracranial carotid artery aneurysms (73\%) were saccular (median size, $11 \mathrm{~mm}$; range, 5.0-38.5 mm), and 4 were fusiform (median size, 21.5 mm; range, 10.0-40.0 $\mathrm{mm}$ ). Eleven of 15 aneurysms (73\%) exhibited gadolinium enhancement at baseline. Four aneurysms (27\%) showed growth at followup imaging, 2 gadolinium-positive $(+)$ and 2 gadolinium-negative $(-)(P=.245)$. Three patients $(21 \%)$ had ipsilateral brain infarcts at baseline; 1 of them showed a new silent brain infarct at follow-up imaging (gadolinium+). Nine patients (64\%) showed bilateral white matter lesions at baseline. In 3 patients, increased white matter lesion severity was observed at follow-up ( 2 gadolinium + ). All observations showed excellent inter- and intraobserver reproducibility.

CONCLUSIONS: In this explorative study, we demonstrated that extracranial carotid artery aneurysm wall imaging was feasible. Future well-powered studies are needed to investigate whether extracranial carotid artery aneurysm gadolinium enhancement predicts aneurysm growth and thromboembolic complications.

ABBREVIATIONS: $\mathrm{ECAA}=$ extracranial carotid artery aneurysm; $\mathrm{Gd}=$ gadolinium; $\mathrm{WML}=$ white matter lesion; $\mathrm{CAR}=$ Carotid Aneurysm Registry

$\mathrm{T}$ he optimal treatment and prognosis of extracranial carotid artery aneurysms (ECAAs) are still unclear. Most ECAAs are coincidental findings, and a conservative approach is presently considered justified in patients with asymptomatic ECAAs. ${ }^{1}$ Several surgical and endovascular techniques have been

Received August 21, 2019; accepted after revision January 7, 2020.

From the Departments of Vascular Surgery (C.J.H.C.M.v.L., M.L.R., V.E.C.P., N.K.N.J., G.J.d.B.) and Radiology (T.L., J.H.), and UMC Utrecht Brain Center (M.D.I.V.), Department of Neurology and Neurosurgery, University Medical Center Utrecht, Utrecht University, Utrecht, the Netherlands.

C.J.H.C.M. van Laarhoven and M.L. Rots contributed equally to this work.

Please address correspondence to Gert J. de Borst, MD, PhD, University Medical Center Utrecht, Department of Vascular Surgery, G04.129 PO Box 85500, 3508 GA Utrecht, the Netherlands; e-mail: g.j.deborst-2@umcutrecht.nl

Indicates article with supplemental on-line tables.

Indicates article with supplemental on-line photos.

http://dx.doi.org/10.3174/ajnr.A6442 developed for ECAA exclusion, but these interventions are mostly reserved for patients with neurologic symptoms or proved growth. ${ }^{2,3}$

Histopathologic research revealed dissection and degeneration as the 2 main causes of ECAAs, with the presence of inflammatory cells in the degenerative vessel wall. ${ }^{4} \mathrm{MR}$ imaging and histopathologic studies of atherosclerotic carotid arteries have suggested that gadolinium (Gd) enhancement reflects the density of the vasa vasorum and inflammation in the arterial wall. ${ }^{5,6}$ Also, MR imaging has been proposed as a noninvasive tool for early detection of arterial wall changes and may be useful for routine monitoring and evaluation of disease activity. ${ }^{6}$ However, ECAA growth pattern and long-term risk of thromboembolization and subsequent stroke have yet to be elucidated.

Previous studies have shown that patients with carotid stenosis have (subclinical) microvascular cerebral damage due to 
microembolic events. ${ }^{7}$ Silent brain infarction and white matter lesions (WMLs) have both been correlated with carotid plaque vulnerability and thus may be a reflection of microembolic events from a proximal source. ${ }^{8,9}$ Silent brain infarcts and WML presence were described to correlate with increased future risk of ischemic stroke and cognitive decline across time. ${ }^{10-12}$ Assessment of both silent and symptomatic brain infarcts and WMLs may, therefore, be helpful surrogate markers for indicating cerebral outcome in patients with ECAAs.

New imaging techniques are required to assess ECAA instability for predicting growth and cerebrovascular outcome. In this study of patients with conservatively treated asymptomatic ECAAs, we aimed to investigate the feasibility of ECAA wall imaging and explored a potential relationship of aneurysm wall enhancement with aneurysm growth and the presence of (silent) brain infarcts and WMLs.

\section{MATERIALS AND METHODS \\ Participants}

Ethics approval for this study was provided by the Medical Research Ethics Committee of the University Medical Center Utrecht, Utrecht, the Netherlands, September 13, 2016 (protocol number 16-341), and all research was conducted according to the principles of the Declaration of Helsinki (59th Amendment, Seoul 2008) and in accordance with the Dutch Medical Research Involving Human Subjects Act. Included patients with an ECAA were selected from the Carotid Aneurysm Registry (CAR; https://www.carotidaneurysmregistry.com/en/home). The registry protocol has been published elsewhere. ${ }^{13}$ In short, any patient 18 years of age or older with an ECAA is included in this ongoing registry, independent of etiology or treatment strategy. Baseline characteristics and follow-up data are collected in a prospective manner. For the present study, we selected asymptomatic patients (no symptoms for at least 1 year before the start of the study) from the CAR with a conservatively treated ECAA. Exclusion criteria were impaired renal function (glomerular filtration rate of $<30 \mathrm{~mL} / \mathrm{min} / 1.73 \mathrm{~m}$ ), Gd contrast allergy, or pregnancy. All selected patients provided informed consent and were recruited between February and September 2017.

\section{MR Imaging}

Included patients were asked to undergo MR imaging at the moment of inclusion and 1 year afterward. Two observers (C.J.H.C.M.v.L., M.L.R.), blinded to patient characteristics and previous imaging, independently scored each of the MR images according to a predefined protocol and case report form. Before scoring, both observers were trained with 2 test scans to familiarize them with the scoring protocol. Test results were compared to overcome interpretation differences. Aneurysm location, shape and size, vessel wall enhancement, and cerebral complications were reported for 2 series of MR images (baseline and follow-up), with a minimum of 2 weeks between the measurements. For a subset of MR images, a third series was re-evaluated after 2 weeks to determine intraobserver variability. For the final data analysis, disagreements were discussed until consensus was reached.
A 3T MR imaging scanner (Ingenia, Software release 5.3; Philips Healthcare, Best, the Netherlands) was used. Images were acquired with an 18-element neurovascular coil as a receiver coil. The MR imaging protocol consisted of a 3D time-of-flight sequence, a contrast-enhanced $\mathrm{T} 13 \mathrm{D}$ sequence (spatial resolution, $0.5 \times 0.5 \times 0.5 \mathrm{~mm}$ ), and, for imaging of the vessel wall, a transverse T1 3D multishot spin-echo sequence of the ipsi- and contralateral extracranial carotid arteries with the following parameters: FOV, $200 \times 166 \times 45 \mathrm{~mm}$ (by default but coverage could be adjusted in case of aneurysms of $>45 \mathrm{~mm}$ ); acquired resolution, $0.5 \times 0.5 \times 1.0 \mathrm{~mm}$; reconstructed resolution, $0.5 \times$ $0.5 \times 0.5 \mathrm{~mm}$; TR/TE, $1.5 / 0.04 \mathrm{~s}$. An anti-driven equilibrium technique and a minimum flip angle of $25^{\circ}$ in the variable flip angle refocusing pulse train were used for increased vessel wall contrast, fat suppression, and flow suppression of blood. ${ }^{14,15}$ This sequence was performed before and after contrast administration, with a duration of $8 \mathrm{~m} 3$ s per sequence. FLAIR and T2 brain imaging series were performed for detection of infarcts and WMLs. Gadobutrol was used as a contrast agent (Gadovist; Bayer Schering Pharma, Berlin, Germany) (0.1 mL/ $\mathrm{kg}$ [1 mmol] per kilogram of body weight); flow rate, $1.0 \mathrm{~mL} / \mathrm{s}$; time between contrast administration and vessel wall imaging, 11 minutes.

\section{Aneurysm Size Assessment}

Aneurysm size was determined on the contrast-enhanced T1weighted images using double oblique multiplanar reconstructions, in which maximum diameters were measured. Diameters were rounded to $0.5 \mathrm{~mm}$. A fusiform or spindle-shaped ECAA was defined as $\geq 150 \%$ dilation of the arterial diameter, compared with the unaffected contralateral carotid artery diameter. In case of bilateral dilation, the diameter of the nonaffected part of the ipsilateral carotid artery was used for comparison. For saccular ECAAs, all sizes were accepted. ${ }^{1}$ In case of a saccular type, the length, width, and neck of the aneurysm were reported; in case of a fusiform type, the length and width were reported (Fig 1). It was decided to define growth as a minimum difference of at least $2.0 \mathrm{~mm}$ measured in at least 1 of the measured dimensions (length, width, and neck), to ensure reliable assessment of growth at the acquired resolution $(0.5 \times 0.5 \times 0.5 \mathrm{~mm})$. We considered differences of $<1.0 \mathrm{~mm}$ in ECAA size to be clinically irrelevant.

\section{Assessment of Gd ECAA Wall Enhancement}

The appearance of the aneurysms was compared before and after administration of Gd to determine the presence of wall enhancement. Aneurysm wall enhancement was considered present if there was a hyperintensity of the vascular wall on MR imaging after Gd administration that was not present on the MR imaging before Gd administration (Fig 2). Ipsilateral aneurysm wall enhancement was reported as well as contralateral enhancement of the internal carotid artery at the level of the aneurysm as a reference.

\section{Assessment of Brain Infarcts and WMLs}

The presence of cortical, subcortical, and lacunar infarcts on the ipsi- or contralateral side was reported $^{16}$ and expressed as 
symptomatic or silent brain infarction. WMLs were semiquantitatively assessed at baseline and follow-up using the Fazekas scale. ${ }^{17,18}$ In short, WMLs were separated according to whether hyperintense lesions were contiguous with the lateral ventricular border (periventricular lesion) or distinct and subcortical (including those in the deep white matter). Subcortical extensions from cortical and lacunar infarcts were excluded.
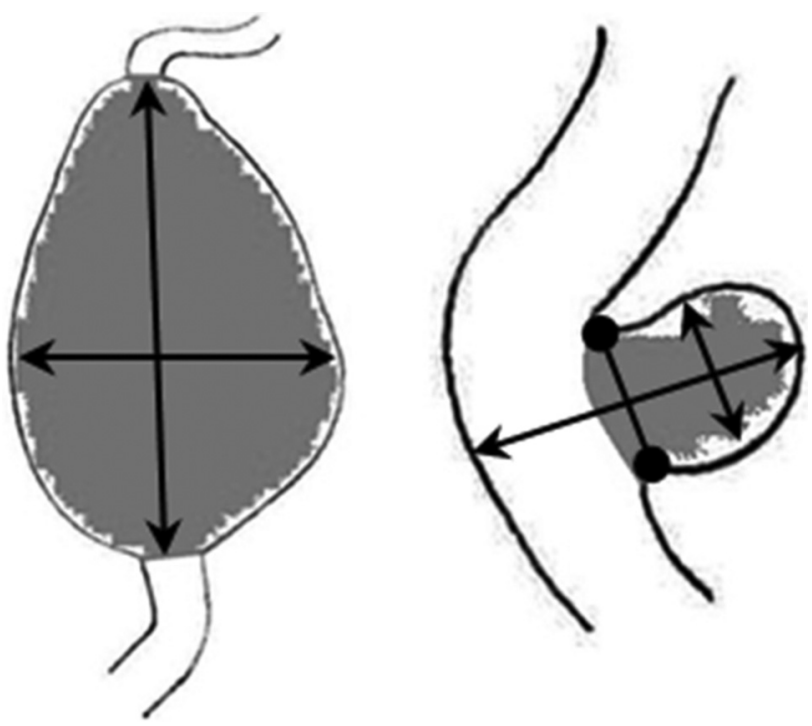

FIG 1. Schematic examples of diameter measurements of ECAAs, perpendicular to the nonaffected vessel. Left: fusiform ECAA length $\times$ width. Right: saccular ECAA length $\times$ width $\times$ neck (round arrow).

\section{Statistical Analysis}

All observations were assessed in terms of reliability and agreement. Inter- and intraobserver reliability of each diameter was calculated by the intraclass correlation coefficient (model: 2-way mixed; type: absolute agreement) with 95\% confidence intervals. Bland-Altman analysis was used to assess agreement for each diameter. For nominal and binary variables (aneurysm location, shape, Gd enhancement, and infarcts), $\kappa$ values were calculated, and for ordinal variables (WMLs), the weighted $\kappa$ was calculated. ${ }^{19}$ The $95 \%$ CIs for $\kappa$ were obtained by use of the psy and boot packages (http://cran.r-project.org) in R Studio, Version 3.4.1, (www.rstudio.com). The proportion of agreement was calculated for both nominal and ordinal variables. ${ }^{20}$ We studied the proportion of patients with aneurysm wall enhancement. Then, we investigated whether aneurysm wall enhancement was associated with signs of instability during follow-up. The primary outcome was the prevalence of aneurysm wall enhancement. Secondary outcomes were growth of the aneurysm sac during follow-up and the presence of WMLs and (silent) brain infarcts at baseline and follow-up. Patients were compared on the basis of Gd enhancement using the Fisher exact test and Mann-Whitney $U$ test, and $P<.05$ was considered statistically significant. These statistical analyses were conducted using SPSS, Version 25.0 (Released 2017; IBM, Armonk, New York).

\section{RESULTS}

Fifteen patients with 16 ECAAs were included and underwent baseline MR imaging. Fourteen patients (with 15 aneurysms) underwent follow-up MR imaging after 1 year. Individual patient characteristics are listed in On-line Table 1. The patient who was
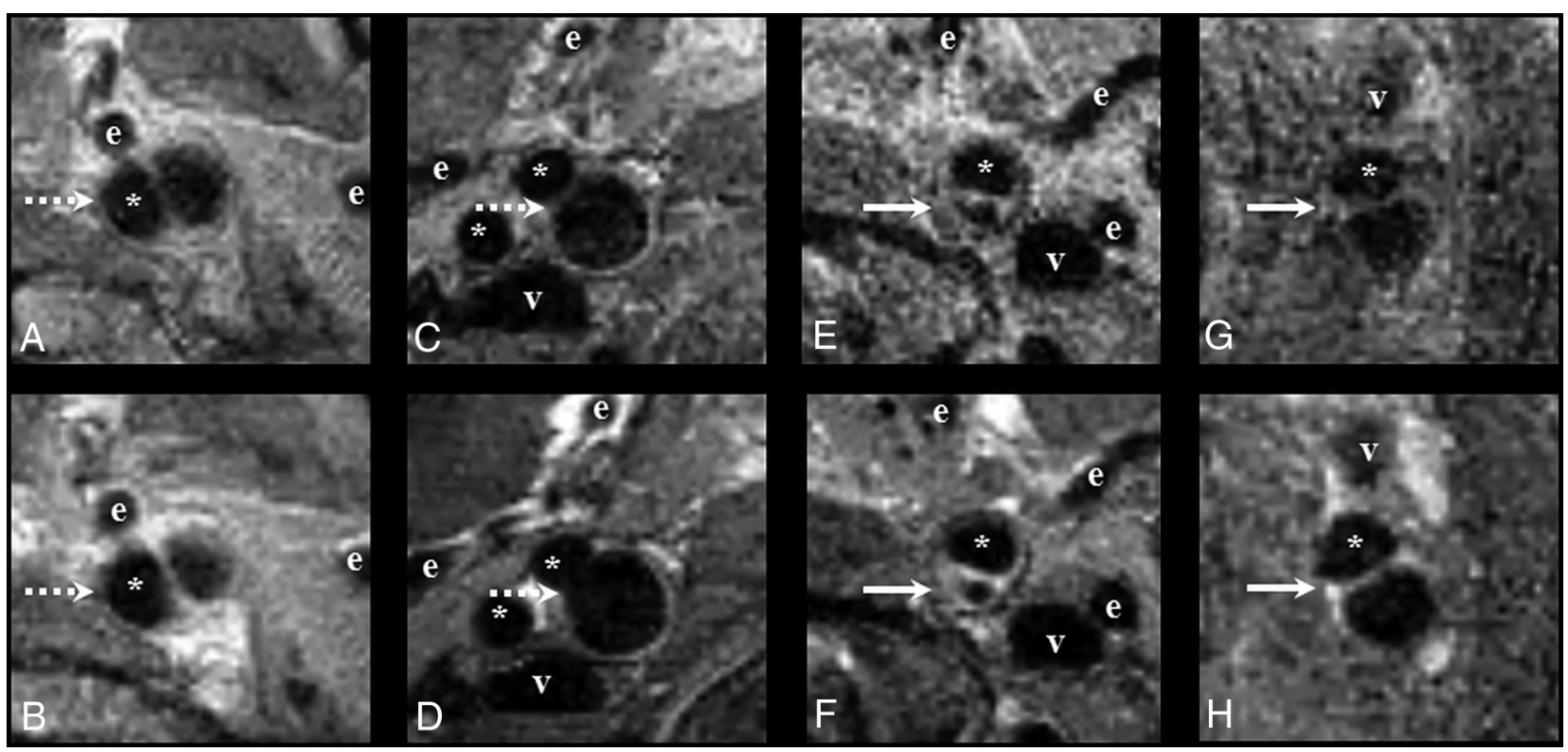

FIG 2. Examples of aneurysm wall enhancement (solid arrow) on $3 T$ MR imaging with aneurysm wall imaging on the transaxial plane. $B, D, F$, and $H$, The sequence after administration of gadolinium. $A$ and $B, A$ 66-year-old woman with a 11.0- mm saccular left internal carotid artery aneurysm without enhancement (dashed arrow). $C$ and D, A 54-year-old man with a 9.0-mm saccular right internal carotid artery aneurysm without enhancement (dashed arrow). $E$ and $F$, A 51-year-old woman with a 7.5-mm saccular left internal carotid artery aneurysm with aneurysm enhancement (solid arrow). $\mathrm{G}$ and $\mathrm{H}$, A 59-year-old woman with a 10.0-mm fusiform left internal carotid artery aneurysm with enhancement (solid arrow). The asterisk indicates the parent ICA; e, branches of the external carotid artery; v, internal jugular vein. 


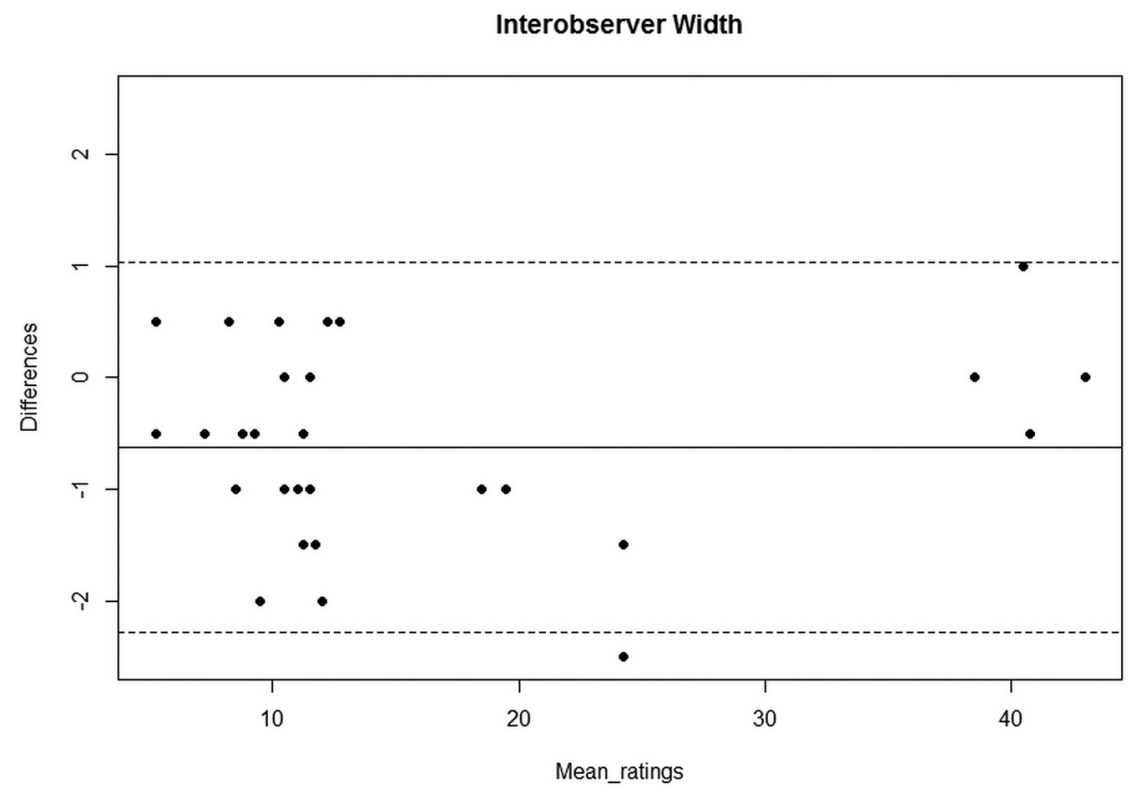

FIG 3. Bland-Altman plot of interobserver agreement. The solid line in the middle represents the mean difference of the diameter measurements between the 2 observers; the dashed line represents the upper and lower limits of agreement (mean difference $\pm 1.96 \times$ SD).

Aneurysm size for aneurysms with and without gadolinium enhancement

\begin{tabular}{|c|c|c|c|}
\hline & No Gadolinium Enhancement $(n=4)$ & Gadolinium Enhancement ( $n=12$ ) & $P$ \\
\hline Length baseline median (range) & $10.75(6.5-46.0)$ & $14.5(7.00-66.5)$ & .316 \\
\hline Width baseline median (range) & $10.5(9.0-41.0)$ & $11.5(5.5-38.5)$ & .953 \\
\hline Neck baseline ${ }^{a}$ median (range) & $9.5(8.0-11.0)$ & $12.0(3.5-22.0)$ & .482 \\
\hline Growth (length) median (range) & $1.0(-1.0-4.0)$ & $0.5(-0.5-4.5)$ & $>.999$ \\
\hline Growth (width) median (range) & $1.0(-1.0-4.0)$ & $0.5(-0.5-4.5)$ & $>.999$ \\
\hline Growth (neck) ${ }^{a}$ median (range) & $0.0(-1.0-0.5)$ & $2.0(0.5-3.0)$ & $.012^{\mathrm{b}}$ \\
\hline Growth (any direction $\geq 2 \mathrm{~mm}$ ) (No.) (\%) & $2(50)$ & $2(16.7)$ & .245 \\
\hline
\end{tabular}

a Only patients with saccular aneurysms.

${ }^{\mathrm{b}} \mathrm{P}<.05$.

lost to follow-up was unable to participate in study activities due to clinical deterioration as a result of a prior cerebral infarction. Eight of 15 patients (53\%) were men. The median age was 55 years (range, 40-69 years). Detailed medical history is summarized in On-line Table 2.

All MR imaging observations showed excellent inter- and intraobserver reproducibility, with both intraclass correlation coefficients and weighted $\kappa>0.9$ and acceptable differences for agreement (Fig 3, On-line Tables 3 and 4, and On-line Figs 1 and 2). In total, 666 observations per observer were made, about which $15 \%$ of disagreements were discussed.

\section{Aneurysm Analysis}

All 16 ECAAs were located in the extracranial internal carotid artery ( 8 on the right, 6 on the left, and 1 patient with bilateral ECAAs). Twelve aneurysms were saccular (75\%); and 4, fusiform. The median length was $13.5 \mathrm{~mm}$ (range, 6.5-66.0 mm), and median width was $11.5 \mathrm{~mm}$ (range, 5.5-41.0 mm). The median neck width was $11 \mathrm{~mm}$ (range, $3.5-22.0 \mathrm{~mm}$ ) in saccular ECAAs. Twelve of 16 (75\%) aneurysms had Gd wall enhancement at baseline. Of the 15 aneurysms with follow-up imaging available, 4 (27\%) had increased in size by $\geq 2.0 \mathrm{~mm}$ in at least 1 direction at the follow-up MR imaging. Of these 4 aneurysms, 1 showed growth in length, width, and neck, 1 showed only increased width, and 2 showed only an increase in length in comparison with baseline. For further analysis, only patients with available follow-up MRA are included.

\section{Aneurysm Wall Gd Enhancement}

All 28 aneurysm wall scans showed good image quality, and no artifacts interfered with our assessment of wall enhancement. Of the 15 aneurysms with follow-up imaging, 11 exhibited ECAA wall enhancement at baseline. Length, width, and neck diameter of aneurysms with wall enhancement compared with those without were similar (Table). Of the 4 aneurysms that increased in size at follow-up, 2 (50\%) showed aneurysm wall Gd enhancement on baseline MR imaging (Table). When absolute differences between baseline and follow-up MR imaging were compared, the median increase in neck size (only saccular aneurysms) was larger in the $8 \mathrm{Gd}$-positive $(+)$ (median, $2.0 \mathrm{~mm}$; range, $0.5-3.0 \mathrm{~mm}$ ) compared with the $3 \mathrm{Gd}$-negative (-) ECAAs (median, $0.0 \mathrm{~mm}$; range, $-1.0-0.5 \mathrm{~mm} ; P=.012$ ). Other differences across time (length and width) were comparable in patients with and without aneurysm wall enhancement (Table). A total of 3 patients 


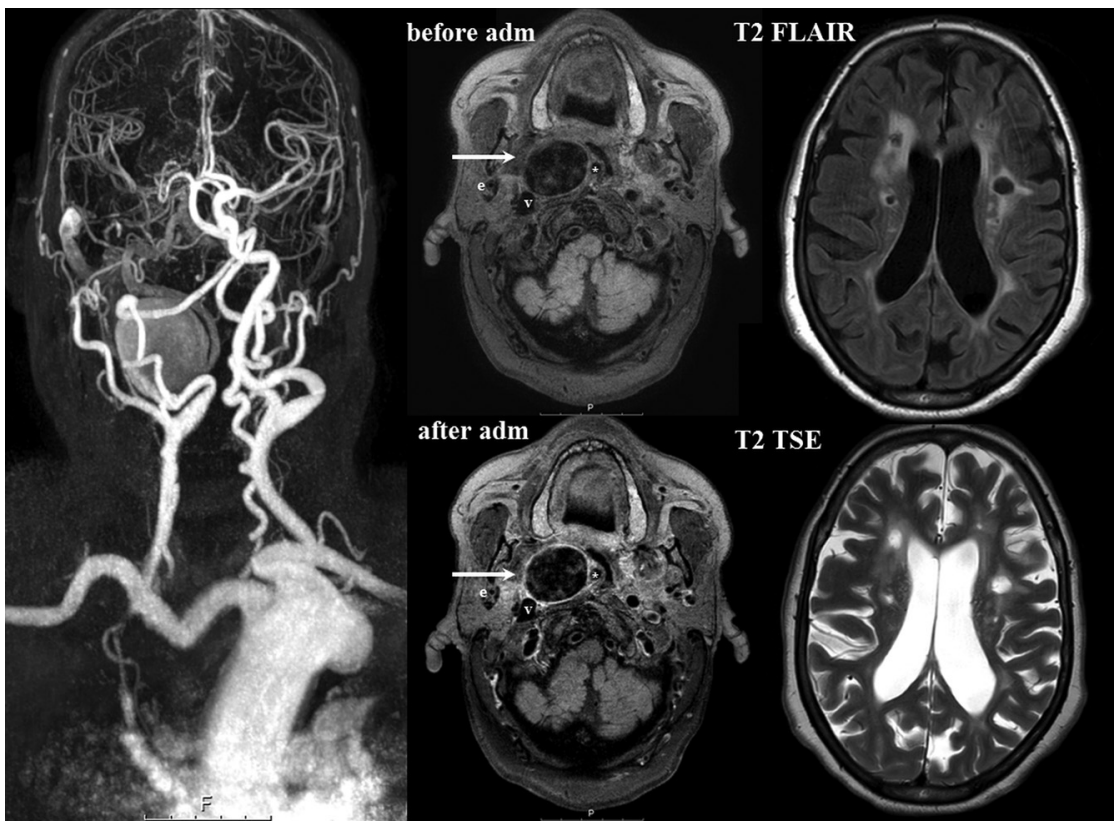

FIG 4. Overview baseline $3 T$ Gd-enhanced MR imaging of 66-year-old woman with a saccular ECAA of the right internal carotid artery. At baseline, enhancement of the aneurysm wall was observed after contrast administration, indicated by the white arrows. T2 FLAIR and T2 TSE show both periventricular and deep white matter lesions and Fazekas 3 and multiple bilateral lacunar infarctions. adm indicates contrast administration.

showed wall enhancement of the contralateral carotid artery at the level of the aneurysm, all of whom also had ipsilateral enhancement of the ECAA wall. On follow-up, 9/15 aneurysms showed steady ipsilateral wall enhancement and $3 / 15$ aneurysms consistently showed no enhancement. One $\mathrm{Gd}+$ aneurysm at baseline no longer showed enhancement at follow-up, and 1 patient with Gd- at baseline showed novel enhancement at follow-up, whereas this was not present at baseline. For the remaining patient, follow-up enhancement could not be evaluated because the scan level was set intracranially.

\section{Assessment of Brain Infarcts and WML}

At baseline, 2 patients showed lacunar (silent) brain infarcts, 1 ipsilateral and 1 with multiple bilateral lacunar brain infarcts (Fig 4). Two other patients had cortical infarcts, 1 of them ipsilateral to the aneurysm and another with a cortical infarct contralateral to the aneurysm. In both of these patients, cortical infarcts were clinically symptomatic, though $>1$ year before the start of the study. No subcortical infarcts were observed, and none of the infarcts were located in posterior circulation. Follow-up MR imaging revealed that the patient who showed multiple bilateral lacunar infarcts at baseline had a bilateral increase in the number of lacunar silent brain infarcts on follow-up imaging. Another patient had 1 new contralateral lacunar silent brain infarct. All patients with infarcts at baseline or follow-up showed Gd enhancement at baseline.

The median Fazekas score at baseline was 1 (range, 0-3) for ipsilateral periventricular WMLs and 1 for contralateral periventricular WMLs (range, $0-3 ; P=.705$ ). The median Fazekas score for deep WMLs was 1 (range, 0-3) at the site ipsilateral to the aneurysm and 0 (range, $0-3$ ) on the contralateral site $(P=.816)$. Three of 14 patients with follow-up imaging showed an increase in the Fazekas score after 1 year, with 2 on the ipsilateral side (1 patient with an increase of deep WMLs and 1 patient with an increase of periventricular WMLs) and 1 patient with a bilateral increase of deep WMLs. Two of 3 patients who showed an increase of WMLs had Gd enhancement at baseline imaging.

\section{DISCUSSION}

In this explorative imaging study, we demonstrated that ECAA wall imaging was feasible, with good interand intraobserver reliability and agreement. Most aneurysms had Gd enhancement of the ECAA wall at baseline. Aneurysm growth occurred in $27 \%$ of all aneurysms.

No earlier studies investigated wall enhancement of extracranial carotid aneurysms. Only patients with Gd enhancement of atherosclerotic plaques in the carotid arteries are described. ${ }^{21-23}$ It is believed that $\mathrm{Gd}$ enhancement reflects both the density of the vasa vasorum and endothelial permeability and can indicate local inflammation of the vessel wall. ${ }^{21-23}$ These previously performed studies used $1.5 \mathrm{~T}^{21,23}$ or $3 \mathrm{~T}^{22} \mathrm{MR}$ imaging scanners, with double molality concentration of Gd and flow rate. The current scanning protocol was based on earlier studies investigating intracranial vessel wall imaging and reporting good image quality and few artifacts. ${ }^{14}$ Fine-tuning of the optimal imaging protocol for the carotid wall specifically, including the sequences used to suppress artifacts due to blood flow, contrast agent concentration, flow rate, and time between administration and vessel wall imaging, is warranted so that uniform reporting standards can be established and study results can be compared.

A follow-up study that included 65 intracranial aneurysms with a median follow-up of 27 months (range, 20-31 months) showed that 4 of 19 aneurysms with wall enhancement at baseline had instability during follow-up, compared with 0 of 46 aneurysms without wall enhancement. ${ }^{24}$ Although our study in patients with ECAAs was not powered to detect differences in aneurysm growth between patients with and without aneurysm wall enhancement, our follow-up results are of interest because we also found aneurysm growth in 2 of 4 patients without wall enhancement. Because healthy intracranial arteries lack an adventitial vasa vasorum, ${ }^{25,26} \mathrm{Gd}$ enhancement of intracranial arteries may indicate stronger local pathologic neovascularization and thus inflammation than in extracranial carotid arteries. ${ }^{26}$ Moreover, the discrimination between moderate or major enhancement in extracranial arteries, possibly indicating pathologic neovascularization of the aneurysm wall instead of 
normal physiology of the vasa vasorum, is quite challenging and subject to the observer's interpretation.

Although acceptable reproducibility of $\mathrm{Gd}+$ was observed in the present study, a Gd+ quantitative measure would accurately increase Gd grading. Density and thus enhancement of the vasa vasorum in normal extracranial arteries is poorly described in the current literature, and between-patient and within-patient variability and its influence on wall enhancement are, at this point, unknown. In contrast to other vascular wall enhancement studies, the present study partly anticipated this issue by also examining the contralateral artery as a reference. Of note, only a minority (3/11) of patients with ipsilateral enhancement also had contralateral enhancement ( 1 of whom had bilateral ECAAs), so although not predictive of aneurysm growth, Gd enhancement does seem to reflect disease activity of the vessel wall.

All 3 patients with ipsilateral brain infarcts showed ECAA wall enhancement, but most patients without infarcts also had aneurysm wall enhancement. Brain infarcts and especially (small) cortical infarcts are often caused by thromboemboli from a proximal source such as the carotid arteries. ${ }^{12}$ Silent brain infarcts have been associated with a 3 -fold increase in the risk of stroke. ${ }^{12}$ In asymptomatic patients with atherosclerotic stenosis of the carotid artery, those with silent brain infarcts benefit more from revascularization procedures in terms of averting stroke risk compared with those without. ${ }^{11}$ Patients with ECAAs at risk of thromboembolic complications may also be identified by assessment of ipsilateral (silent) brain infarcts, in addition to assessment of ECAA wall Gd enhancement or aneurysm growth. Structural imaging of the brain may contribute to clinical decision-making on whether to treat (eg, conservatively or surgically) these patients.

We found that two-thirds of patients had bilateral WMLs. Because there was no clear asymmetry and the presence of WMLs did not seem restricted to patients with ECAA wall enhancement, on the basis of our preliminary results, WMLs do not seem directly related to Gd enhancement of the aneurysm wall. Previous studies have shown that the cause of WMLs is multifactorial, and thrombo-embolism from a proximal source is only one of the potential causes of these radiographic findings. ${ }^{8,27}$ It is striking, however, that these relatively young patients included in our study (median age, 54 years) very often showed a high (bilateral) WML score compared with literature investigating WMLs in an otherwise healthy population. ${ }^{28}$ WMLs have been used widely as a surrogate marker for small-vessel disease and have been closely associated with risk factors for atherosclerotic disease such as hypertension. ${ }^{29}$ These findings suggest that not only a causal relationship exists between the presence of ECAAs and WMLs but also a shared etiology for the presence of both diseases. WMLs have been associated with an increased risk of future stroke ${ }^{30}$ and cognitive decline. ${ }^{29}$ Perhaps medical therapy in patients with ECAAs should be optimized to treat not only possible consequences of thromboemboli but also risk factors underlying generalized vascular disease.

\section{Strengths and Limitations}

The primary aim of our study was feasibility. Because of the small sample size, no conclusions can be drawn regarding the predictive value of wall enhancement. Although good inter- and intraobserver reliabilities were found in our study, assessing the presence of WMLs by means of the Fazekas scale can be highly dependent on expertise and experience. Also, the Fazekas scale is insensitive to subtle lesions and subtle changes across time. Other quantitative or semiquantitative measures, such as the rating scale for age-related white matter changes or computerized assessment of lesion volume, may be more reliable and sensitive to small changes across time. Future studies should include quantitative methods for determining white matter lesion volume so that subtle changes can be detected in an objective manner. Nevertheless, our method is easily translated to clinical practice and can be straightforwardly applied on frequently used MR images (FLAIR and T2). Little is known yet regarding the reliability of using MR imaging for ECAA imaging compared with CT. Although high correlations between the 2 were found in studies on other types of aneurysms, ${ }^{31}$ comparison of these 2 imaging techniques should be a topic for future research.

The study sample is a reflection of clinical practice in ECAA disease; as a consequence, various shapes and sizes of aneurysms with different blood flow characteristics were included. Slow blood flow within the aneurysm sac could cause pseudoenhancement, which potentially affects the validity and clinical applicability of our results. Future computational hemodynamic and histologic studies of aneurysm wall tissue should further elucidate the relation of Gd enhancement and aneurysm wall instability. Last, one might argue that conventional diameter measurements fail to indicate ECAA growth. Because appropriate imaging tools to measure geometric differences are currently lacking, we might have missed cases that expanded in other directions than obliquely.

\section{CONCLUSIONS}

In this small exploratory study, we demonstrated that extracranial carotid artery aneurysm wall imaging was feasible and Gd wall enhancement was identified in most aneurysms. Aneurysm growth was relatively common in a follow-up period of 1 year. Larger prospective studies are warranted to investigate the relation between ECAA wall enhancement and aneurysm growth, (silent) brain infarcts, and white matter lesions.

Disclosures: Tim Leiner-UNRELATED: Grants/Grants Pending: Philips Healthcare, Pie Medical Imaging*; Payment for Lectures Including Service on Speakers Bureaus: Philips Healthcare, Bayer HealthCare Pharmaceuticals*; Patents (Planned, Pending or Issued): inventor, US Patent 10,176,575.* *Money paid to the institution.

\section{REFERENCES}

1. Pourier VE, Welleweerd JC, Kappelle LJ, et al. Experience of a single center in the conservative approach of 20 consecutive cases of asymptomatic extracranial carotid artery aneurysms. Eur J Neurol 2018;25:1285-89 CrossRef Medline

2. Welleweerd JC, Moll FL, De Borst GJ. Technical options for the treatment of extracranial carotid aneurysms. Expert Rev Cardiovasc Ther 2012;10:925-31 CrossRef Medline

3. Welleweerd JC, Den Ruijter HM, Nelissen BG, et al. Management of extracranial carotid artery aneurysm. Eur J Vasc Endovasc Surg 2015;50:141-47 CrossRef Medline 
4. Welleweerd JC, Nelissen BG, Koole D, et al. Histological analysis of extracranial carotid artery aneurysms. PLoS One 2015;10:e11791519 CrossRef

5. Backes D, Hendrikse J, van der Schaaf I, et al. Determinants of gadolinium-enhancement of the aneurysm wall in unruptured intracranial aneurysms. Neurosurgery 2018;83:719-25 CrossRef Medline

6. Oppenheim C, Naggara O, Touzé E, et al. High-resolution MR imaging of the cervical arterial wall: what the radiologist needs to know. Radiographics 2009;29:1413-31 CrossRef Medline

7. Jayasooriya G, Thapar A, Shalhoub J, et al. Silent cerebral events in asymptomatic carotid stenosis. J Vasc Surg 2011;54:227-36 CrossRef Medline

8. van den Bouwhuijsen QJ, Vernooij MW, Verhaaren BFJ, et al. Carotid plaque morphology and ischemic vascular brain disease on MRI. AJNR Am J Neuroradiol 2017;38:1776-82 CrossRef Medline

9. Altaf N, Morgan PS, Moody A, et al. Hyperintensities are associated with carotid intraplaque hemorrhage. Radiology 2008;248:202-09 CrossRef

10. Gensicke H, Van Der Worp HB, Nederkoorn PJ, et al; ICSS-MRI Substudy Investigators. Ischemic brain lesions after carotid artery stenting increase future cerebrovascular risk. J Am Coll Cardiol 2015;65:521-29 CrossRef Medline

11. Streifler JY, den Hartog AG, Pan S, et al; ACST-1 trial Collaborators. Ten-year risk of stroke in patients with previous cerebral infarction and the impact of carotid surgery in the Asymptomatic Carotid Surgery Trial. Int J Stroke 2016;11:1020-27 CrossRef Medline

12. Vermeer SE, Prins ND, den Heijer T, et al. Silent brain infarcts and the risk of dementia and cognitive decline. $N$ Engl J Med 2003; 348:1215-22 CrossRef Medline

13. Welleweerd JC, Bots ML, Kappelle LJ, et al. Rationale and design of the extracranial Carotid artery Aneurysm Registry (CAR). $J$ Cardiovasc Surg (Torino) 2018;59:692-98 CrossRef Medline

14. Dieleman N, Yang W, Van Der Kolk AG, et al. Qualitative evaluation of a high-resolution 3D multi-sequence intracranial vessel wall protocol at 3 Tesla MRI. PLoS One 2016;11:e016078111 CrossRef

15. Yang H, Zhang X, Qin Q, et al. Improved cerebrospinal fluid suppression for intracranial vessel wall MRI. J Magn Reson Imaging 2016;44:665-72 CrossRef Medline

16. de Leeuw F, de Groot J, Bots M, et al. Carotid atherosclerosis and cerebral white matter lesions in a population based magnetic resonance imaging study. J Neurol 2000;247:291-96 CrossRef Medline

17. Fazekas F, Chawluk JB, Alavi A, et al. MR signal abnormalities at 1.5-T in Alzheimer dementia and normal aging. AJR Am J Roentgenol 1987;149:351-56 CrossRef Medline
18. Kim KW, MacFall JR, Payne ME. Classification of white matter lesions on magnetic resonance imaging in the elderly. Biol Psychiatry 2008;64:273-80 CrossRef Medline

19. Kottner J, Audige L, Brorson S, et al. Guidelines for Reporting Reliability and Agreement Studies (GRRAS) were proposed. Int $J$ Nurs Stud 2011;48:661-71 CrossRef Medline

20. de Vet HC, Terwee CB, Knol DL, et al. When to use agreement versus reliability measures. J Clin Epidemiol 2006;59:1033-39 CrossRef Medline

21. Aoki S, Aoki K, Ohsawa S, et al. Dynamic MR imaging of the carotid wall. J Magn Reson Imaging 1999;9:420-27 CrossRef Medline

22. Qiao Y, Etesami M, Astor BC, et al. Carotid plaque neovascularization and hemorrhage detected by MR imaging are associated with recent cerebrovascular ischemic events. AJNR Am J Neuroradiol 2012;33:755-60 CrossRef Medline

23. Kerwin WS, Brien KD, Ferguson MS, et al. Inflammation in carotid atherosclerotic plaque: a dynamic contrast-enhanced MR imaging study. Radiology 2006;241:459-68 CrossRef Medline

24. Vergouwen MD, Backes D, van der Schaaf IC, et al. Gadolinium enhancement of the aneurysm wall in unruptured intracranial aneurysms is associated with an increased risk of aneurysm instability: a follow-up study. AJNR Am J Neuroradiol 2019;40:111-16 CrossRef Medline

25. Krings T, Mandell DM, Kiehl TR, et al. Intracranial aneurysms: from vessel wall pathology to therapeutic approach. Nat Rev Neurol 2011;7:547-59 CrossRef Medline

26. Portanova A, Hakakian N, Mikulis DJ, et al. Vasa vasorum 2013 insights and implications for imaging. Radiology 2013;267:667-79 CrossRef Medline

27. Potter GM, Doubal FN, Jackson CA, et al. Lack of association of white matter lesions with ipsilateral carotid artery stenosis. Cerebrovasc Dis 2012;33:378-84 CrossRef Medline

28. Leeuw FE, De Groot JC, De Achten E, et al. Prevalence of cerebral white matter lesions in elderly people: a population based magnetic resonance imaging study-the Rotterdam Scan Study. J Neurol Neurosurg Psychiatry 2001;70:9-14 Medline

29. Van Dijk EJ, Prins ND, Vrooman HA, et al. Progression of cerebral small vessel disease in relation to risk factors and cognitive consequences: Rotterdam Scan Study. Stroke 2008;39:2712-19 CrossRef Medline

30. Streifler JY, Eliasziw M, Benavente OR, et al; North American Symptomatic Carotid Endarterectomy Trial Group. Prognostic importance of leukoaraiosis in patients with symptomatic internal carotid artery stenosis. Stroke 2002;33:1651-55 CrossRef Medline

31. Acar T, Karakas AB, Ozer MA, et al. Building three-dimensional intracranial aneurysm models from 3D-TOF MRA: a validation study. J Digit Imaging 2019;32:963-70 CrossRef Medline 